\title{
EFFECT OF STORAGE CONDITIONS ON QUALITY OF A FUNCTIONAL POWDER OF CAPE GOOSEBERRY OBTAINED BY SPRAY DRYING
}

\section{EFECTO DEL ALMACENAMIENTO EN LA CALIDAD DE UN POLVO FUNCIONAL DE UCHUVA OBTENIDO POR SECADO POR ASPERSIÓN}

\author{
Gustavo R. Hernández-Sandoval ${ }^{1}$, Misael Cortés-Rodríguez ${ }^{2}$, Héctor J. Ciro-Velásquez *3
}

${ }^{1}$ Food Engineer, M.Sc. gustavohernandezs@hotmail.com; ${ }^{2}$ Professor, Ph.D., Universidad Nacional de Colombia, Medellín, Colombia, mcortesro@unal.edu.co; ${ }^{3}$ Associate Profesor, M.Sc., Ph.D., Universidad Nacional de Colombia, Medellín, Colombia. *Author for correspondence: hjciro@unal.edu.co

Rev. U.D.C.A Act. \& Div. Cient. 17(1): 139-149, Enero-Junio, 2014

\section{SUMMARY}

In Colombia the industrial use of cape gooseberry is poor, which is proposed to evaluate a transformation process leading to a value added product. Functional foods are products that by virtue of their physiologically active components provide health benefits beyond basic nutrition. This research focused on the evaluation during storage of cape gooseberry powder obtained by spray drying and added with vitamin $\mathrm{C}$, iron (ferrous bisglycinate), folic acid, soluble fiber (polydextrose) and soy protein. The powder obtained from the optimization of the process was stored under two different packaging conditions: with vacuum (V) and without vacuum (NV), storage time $(0,1,2,3,4$, 5 and 6 months) and three storage temperatures of 4 , 20 and $30^{\circ} \mathrm{C}$. The results indicated that the best storage condition was packaging without vacuum at a temperature of $20^{\circ} \mathrm{C}$, reaching values at the end of the storage period (six months) of vitamin C, B 9 and iron expressed in $\mathrm{mg} / 100 \mathrm{~g}$ of cape gooseberry powder of $22.33 \pm 1.82,261.74 \pm 5.45$ and $8.88 \pm 0.61$, respectively. Additionally, the protein and dietary fiber in $\mathrm{g} / 100 \mathrm{~g}$ cape gooseberry powder were $6.89 \pm$ 0.27 and $10.44 \pm 0.29$, respectively. A diminishing trend was observed in the color parameters (CIE-Lab) during storage time being more distinct the higher the storage temperature.

Key words: Physalis peruviana L., functional foods, drying, shelf life.

\section{RESUMEN}

En Colombia el aprovechamiento industrial de la uchuva es deficiente por lo cual se propone evaluar un proceso de transformación que otorgue un valor adicional al producto. Alimentos funcionales son productos que por virtud de sus componentes activos proveen beneficios a la salud más allá de componente nutricional. Este trabajo de investigación consistió en la evaluación durante el almacenamiento de polvo de uchuva (Physalis peruviana L.) obtenido mediante secado por aspersión y adicionado con vitamina C, hierro, ácido fólico, fibra soluble (polidextrosa) y proteína de soya. El polvo obtenido a partir de la optimización del proceso de secado por aspersión se almacenó bajo dos condiciones de envasado diferentes: con vacío (V) y sin vacío (NV), para diferentes tiempos de almacenamiento $(0,1,2,3$, 4,5 y 6 meses) y a tres temperaturas de almacenamiento de 4,20 y $30^{\circ} \mathrm{C}$. Los resultados indicaron que la mejor condición de almacenamiento es envasado sin vacío a una temperatura de $20^{\circ} \mathrm{C}$, alcanzando valores al final del período de almacenamiento (6 meses) para la vitamina C, B9 y hierro en $\mathrm{mg} / 100 \mathrm{~g}$ de polvo de uchuva de $22,33 \pm 1,82$; $261,74 \pm 5,45$ y $8,88 \pm 0,61$, respectivamente. Además, la fibra y la proteína dietética en $\mathrm{g} / 100 \mathrm{~g}$ de polvo de uchuva fue de 6,89 $\pm 0,27$ y 10,44 $\pm 0,29$, respectivamente. Una tendencia a la baja se observó en los parámetros de color (CIE-Lab) durante el tiempo de almacenamiento siendo más significativa cuando la temperatura de almacenamiento se incrementó.

Palabras clave: Physalis peruviana L, alimento funcional, secado, vida útil. 


\section{INTRODUCTION}

Cape gooseberry is a tropical fruit found inside a husk that entirely covers and protects it from the surroundings during the stages of harvest and post-harvest (Rojas et al. 2004). It is grown at altitudes within 2300 and 3000 meters above sea level, temperatures between 8 and $17^{\circ} \mathrm{C}$ with a range of relative humidity from 80 to $90 \%$ (Mazorra et al. 2006). This fruit possesses a high content of sugar and vitamins like A, B and C. Additionally, medicinal properties have been attributed (Rodríguez \& Elika, 2007; Puente et al. 2011). When ripe, it presents the following physical-chemical characteristics: $\mathrm{pH}$ of 3.7, ${ }^{\circ}$ Brix between 9 and 13, and percentage of acidity from 1.6 to 2.0 (Osorio \& Roldan, 2004). This set of attributes has made cape gooseberry one of the fruits with the largest exports of Colombia, and with high potential for use as functional food.

Currently there are four methodologies for obtaining functional foods: genetic engineering (Xudong et al. 2000; Prescha et al. 2003) breeding and cropping techniques (Dhirman et al. 1999), matrix engineering (Peña et al. 2013; Cortés, 2012) and bulk incorporation (Pérez et al. 2006). This last technique is industrially the most used where components such as vitamins, minerals, pre and probiotics and essential fatty acids, among others are directly incorporated. This also adds synergic effect on the original food.

Physiological Active Components (PAC) have been used in the fortification of foods. Iron is a fundamental micronutrient in our diets, especially under specific conditions such as menstruations, pregnancy and breastfeeding and its lack produces anemia. Its absorption can be increased by means of promoters such as vitamin C (Gibson, 1997) which is a frequently used antioxidant in the food industry for reducing characteristics of its two groups of protons donors (Koshizaka et al. 1988). Folic acid or vitamin $\mathrm{B}_{9}$ is a hydrosoluble vitamin necessary for the formation of structural proteins and hemoglobin, and which absorption and stability is also promoted by vitamin C (Koshizaka et al. 1988). Polydextrose is a soluble fiber that is not digested in the small intestine, but undergoes a partial fermentation in the large intestine (Ribeiro et al. 2003) representing health benefits since it favors the growth of beneficial bacteria (prebiotics) softening and facilitating fecal matter, reducing $\mathrm{pH}$ and gas production, attenuating blood glucose, and increasing calcium absorption (Pronczuk \& Hayes, 2006).

Spray drying is an effective process for the conservation of food products by reducing aw (water activity,) generating good stability and suitable quality attributes. By means of this process, the liquid feeding is dispersed in the form of fine drops with a high surface area, in such manner that upon direct contact with hot air it favors the transfer of heat and mass and finally obtaining a powder product (Kudra \& Mujumdar, 2012).

Fruits have high contents of soluble solids with low glass transition temperatures $\left(T_{g}\right)$. Thus spray drying is a difficult and complex process and therefore additives are used to avoid product plastification inside the dryer, to increase process performance, to protect active ingredients from oxidation and other undesired reactions and to improve sensory and nutritional characteristics (Bhandari et al. 1993; Dolinsky et al. 2000; Fazaeli et al. 2012).

Powder products are solid materials with very distinct characteristics depending on their nature. During storage, these products can experience a series of changes in its amorphous state from a product initially in vitreous state going to a gummy state, where all properties (physical, physicalchemical, organoleptic, microbiological and nutritional) are substantially modified because of the greater water mobility in the food substrate (Dolinsky et al. 2000; Woun et al. 2012)

The aim of this work was to evaluate the effects of storage conditions: temperature $\left(4^{\circ} \mathrm{C}, 20^{\circ} \mathrm{C}\right.$ and $\left.30^{\circ} \mathrm{C}\right)$, packaging (with and without vacuum) and storage time $(0,1,2,3,4,5$ and 6 months) upon the quality attributes of cape gooseberry powder added with vitamin $\mathrm{C}$, iron, folic acid, soluble fiber (polydextrose) and soy protein.

\section{MATERIALS AND METHODS}

Fresh cape gooseberries were selected from a commercial crop with the following characteristics: free of external damage and with a commercial ripeness in the color scale from 3 to 4 , according to the Colombian Technical Standard NTC 4580 (ICONTEC, 1999). The pulp was obtained by homogenization (ULTRA-TURRAX T25) at 8000 RPM for 2 minutes, crushing the fruit with seeds and skin, and then filtered via a screen of $500 \mu \mathrm{m}$. The mixing pulp was done by homogenization at 20000 RPM for 5 minutes with maltodextrin with dextrose equivalent (DE) of $19-20$ (24.37\%), vitamin C (0.0158\%), folic acid $(0.0001 \%)$, iron $(0.0035 \%)$, polydextrose $(1.38 \%)$ and isolated soy protein $(2.57 \%)$.

A spray dryer with an atomizer disk (Vibrasec S.A.) was used with an evaporation capacity of $1.5 \mathrm{~L} / \mathrm{h}$ under the following operation conditions: inlet air temperature $=194.2^{\circ} \mathrm{C}$, outlet air temperature $=87.7^{\circ} \mathrm{C}$, disk rotation speed $=19848 \mathrm{rpm}$, and vacuum pressure in drying chamber $=0.124 \mathrm{kPa}(0.5$ inches of water).

For the storage study the following variables were established: temperature $\left(4,20\right.$ and $\left.30^{\circ} \mathrm{C}\right)$, packaging conditions with vacuum (V) and without vacuum (NV) and storage times $(0,1,2,3,4,5$ and 6 months). A low density polyethylene 
package was used with high barrier to water vapor and hot sealing. Local barometric pressure was $85 \mathrm{kPa}$ and package vacuum pressure was $-63.75 \mathrm{kPa}$.

Characterization of the cape gooseberry powder: Moisture content $\left(\mathrm{X}_{\mathrm{w}}\right)$ was established by the thermogravimetric method according to standards AOAC 7.003-84 and 930.15-90, adapted from AOAC 934.06 (AOAC, 1996); water activity (aw) was defined with a dew point hygrometer at $25^{\circ} \mathrm{C}$ with equipment AquaLAB Decagon series 3TE according to Standard AOAC 978.18M (AOAC, 1996); pH with a potentiometer Schott CG840B, according to Standard AOAC 981.12 (AOAC, 1990); the color was determined using a spectrophotometer (X-Rite, model SP64,) with illuminant $D_{65}$ and observer of $10^{\circ}$ as reference; color coordinates CIE-Lab, and chromatic coordinates tone (h) and chroma (C) were calculated from the spectrum of reflection of the samples; dietary fiber contents were evaluated by the AOAC 993.21 method (AOAC, 1998) and proteins were determined by the Kjeldahl method according to standard NTC 4657 (ICONTEC, 1999).

PAC: Extraction and Quantification: Vitamin C extraction was performed by diluting the sample in distilled water until complete hydration and then filtered at $4.5 \mu \mathrm{m}$. Quantification was established through High Performance Liquid Chromatography (HPLC) provided with UV/ VIS detector with diode array and detection at 265nm (Gutiérrez et al. 2007). Folic acid was extracted based in the methodology suggested by Arcot \& Shrestha (2005) with sample hydrolysis of $\mathrm{NaOH} 2 \mathrm{M}$ in magnetic agitation, followed by filtration and neutralization with phosphate buffer $\mathrm{pH}$ 3.0. Folic acid determination was performed by HPLC with detection at 280nm (Heudi et al. 2005; Pérez et al. 2006). Iron was determined by the method of atomic absorption spectrophotometry with flame, based in Colombian Technical
Standard (NTC-4807) for the determination of minerals to fruits and vegetables (ICONTEC, 2000)

Statistical Analysis: A factorial experimental design with the factors temperature and storage time was applied evaluating the response variables to the packaging conditions with and without vacuum. The results were analyzed by Design Expert 6.0 software by means of response surfaces methodology. The different response variables were adjusted to the following second degree polynomial model:

$$
Y=b_{o}+\sum_{i=1}^{k} b_{i} X_{i}+\sum_{i=1}^{k} b_{i i} X_{i}^{2}+\sum_{i=1}^{k-1} \sum_{\substack{j=2 \\ j>i}}^{k} b_{i j} X_{i} X_{j}
$$

Where $b_{i}$ represent adjustment constants, $X_{i}$ represents statistical model factors $(A=$ storage time; $B=$ Storage temperature) and $\mathrm{k}$ is the number of factors in the model.

\section{RESULTS AND DISCUSSION}

PAC contents in cape gooseberry powder: Tables 1 and 2 present the mean values and standard deviation obtained to the response variables evaluated during the storage of the product under the packaging conditions: without vacuum (NV) and vacuum (V). Additionally, the results of Analysis of Variance $(\alpha=0.05)$ are shown in the Table 3.

Figure 1 presents the results of the response surface of the contents of vitamin $\mathrm{C}, \mathrm{B}_{9}$, and iron in $100 \mathrm{~g}$ of cape gooseberry powder in function of the storage factors such as temperature, storage time and packaging conditions. It was observed that packaging condition was not a determinant. This fact can be attributable to the encapsulation of the PAC with the maltodextrin (Saénz et al. 2009; Ersus \& Yurdagel, 2007).

Table 1. Experiment results in storage without vacuum (NV).

\begin{tabular}{|c|c|c|c|c|c|c|c|c|c|c|c|}
\hline $\begin{array}{c}\text { Temperature } \\
{ }^{\circ} \mathrm{C}\end{array}$ & $\begin{array}{l}\text { Time } \\
\text { month }\end{array}$ & $\begin{array}{c}\text { Vitamin C } \\
\mathrm{mg} / 100 \mathrm{~g}\end{array}$ & $\begin{array}{l}\text { Folic Acid } \\
\mathrm{mcg} / 100 \mathrm{~g}\end{array}$ & $\begin{array}{c}\text { Iron } \\
\mathrm{mg} / 100 \mathrm{~g}\end{array}$ & $\begin{array}{c}\text { Dietary fiber } \\
\mathrm{g} / \mathbf{1 0 0 \mathrm { g }}\end{array}$ & $\begin{array}{l}\text { Protein } \\
\mathrm{g} / 100 \mathrm{~g}\end{array}$ & $a_{w}$ & $\mathrm{pH}$ & $\mathrm{L}^{*}$ & $a^{*}$ & $b^{*}$ \\
\hline \multirow[t]{7}{*}{4} & 0 & $36.68 \pm 0.05$ & $276.92 \pm 3.02$ & $10.26 \pm 0.20$ & $10.58 \pm 1.05$ & $6.97 \pm 1.63$ & $0.277 \pm 0.005$ & $3.65 \pm 0.03$ & $81.25 \pm 0.15$ & $4.21 \pm 0.04$ & $22.59 \pm 0.07$ \\
\hline & 1 & $36.57 \pm 0.31$ & $277.26 \pm 5.82$ & $10.12 \pm 0.16$ & $10.34 \pm 1.36$ & $7.04 \pm 1.35$ & $0.325 \pm 0.002$ & $3.45 \pm 0.02$ & $80.44 \pm 0.20$ & $4.24 \pm 0.13$ & $22.43 \pm 0.31$ \\
\hline & 2 & $30.66 \pm 1.09$ & $274.04 \pm 4.72$ & $8.87 \pm 0.21$ & $10.7 \pm 1.95$ & $6.52 \pm 1.2$ & $0.334 \pm 0.002$ & $3.59 \pm 0.08$ & $79.76 \pm 0.11$ & $4.27 \pm 0.05$ & $21.18 \pm 0.02$ \\
\hline & 3 & $27.99 \pm 0.70$ & $274.30 \pm 6.30$ & $9.07 \pm 0.29$ & $10.97 \pm 2.64$ & $6.98 \pm 2.04$ & $0.347 \pm 0.002$ & $3.56 \pm 0.05$ & $80.12 \pm 0.71$ & $4.42 \pm 0.04$ & $21.18 \pm 0.04$ \\
\hline & 4 & $26.68 \pm 0.77$ & $272.03 \pm 7.29$ & $11.13 \pm 0.54$ & $10.48 \pm 1.71$ & $7.15 \pm 2.01$ & $0.362 \pm 0.002$ & $3.63 \pm 0.03$ & $80.04 \pm 0.29$ & $4.65 \pm 0.04$ & $21.56 \pm 0.28$ \\
\hline & 5 & $25.22 \pm 0.31$ & $269.74 \pm 5.48$ & $9.00 \pm 0.71$ & $10.18 \pm 0.63$ & $7.06 \pm 0.93$ & $0.356 \pm 0.003$ & $3.47 \pm 0.06$ & $79.31 \pm 0.70$ & $4.65 \pm 0.04$ & $22.38 \pm 0.29$ \\
\hline & 6 & $25.64 \pm 1.62$ & $270.92 \pm 2.50$ & $8.47 \pm 0.17$ & $10.23 \pm 0.99$ & $6.49 \pm 0.77$ & $0.360 \pm 0.003$ & $3.68 \pm 0.03$ & $79.63 \pm 0.29$ & $4.68 \pm 0.03$ & $21.14 \pm 0.27$ \\
\hline
\end{tabular}


Continuation Table 1.

\begin{tabular}{|c|c|c|c|c|c|c|c|c|c|c|c|}
\hline \multirow{7}{*}{20} & 0 & $36.68 \pm 0.05$ & $276.92 \pm 3.02$ & $10.26 \pm 0.20$ & $10.11 \pm 1.7$ & $6.69 \pm 0.74$ & $0.277 \pm 0.005$ & $3.55 \pm 0.04$ & $81.25 \pm 0.15$ & $4.21 \pm 0.04$ & $22.59 \pm 0.07$ \\
\hline & 1 & $29.29 \pm 0.91$ & $272.43 \pm 5.07$ & $10.22 \pm 1.19$ & $10.98 \pm 0.29$ & $7.2 \pm 1.46$ & $0.360 \pm 0.002$ & $3.67 \pm 0.05$ & $82.27 \pm 0.57$ & $4.47 \pm 0.06$ & $22.05 \pm 0.26$ \\
\hline & 2 & $25.30 \pm 1.71$ & $270.88 \pm 9.02$ & $9.27 \pm 0.21$ & $10.98 \pm 1.97$ & $7.05 \pm 1.14$ & $0.367 \pm 0.001$ & $3.51 \pm 0.09$ & $80.44 \pm 0.21$ & $5.04 \pm 0.04$ & $23.02 \pm 0.05$ \\
\hline & 3 & $24.16 \pm 1.84$ & $268.61 \pm 11.50$ & $8.37 \pm 0.46$ & $10.1 \pm 1.2$ & $7.08 \pm 2.09$ & $0.383 \pm 0.002$ & $3.42 \pm 0.08$ & $79.34 \pm 0.06$ & $5.04 \pm 0.02$ & $21.54 \pm 0.08$ \\
\hline & 4 & $23.01 \pm 1.18$ & $265.09 \pm 2.99$ & $11.90 \pm 0.65$ & $10.22 \pm 0.62$ & $6.61 \pm 1.66$ & $0.394 \pm 0.003$ & $3.61 \pm 0.02$ & $80.16 \pm 0.03$ & $5.20 \pm 0.03$ & $19.97 \pm 0.05$ \\
\hline & 5 & $21.92 \pm 0.80$ & $263.33 \pm 6.06$ & $8.50 \pm 0.14$ & $10.27 \pm 1.99$ & $7.07 \pm 1.13$ & $0.386 \pm 0.006$ & $3.63 \pm 0.02$ & $80.44 \pm 0.29$ & $5.29 \pm 0.02$ & $19.89 \pm 0.26$ \\
\hline & 6 & $21.78 \pm 2.05$ & $260.25 \pm 2.83$ & $8.90 \pm 0.82$ & $10.2 \pm 0.54$ & $6.8 \pm 0.81$ & $0.392 \pm 0.002$ & $3.52 \pm 0.04$ & $79.63 \pm 0.29$ & $5.33 \pm 0.03$ & $19.07 \pm 0.24$ \\
\hline \multirow{7}{*}{30} & 0 & $36.68 \pm 0.05$ & $276.92 \pm 3.02$ & $10.26 \pm 0.20$ & $10.5 \pm 2.05$ & $6.96 \pm 1.21$ & $0.277 \pm 0.005$ & $3.6 \pm 0.04$ & $81.25 \pm 0.15$ & $4.21 \pm 0.04$ & $22.59 \pm 0.07$ \\
\hline & 1 & $25.19 \pm 1.62$ & $267.73 \pm 6.87$ & $10.83 \pm 0.72$ & $10.74 \pm 2.54$ & $7.18 \pm 0.53$ & $0.333 \pm 0.002$ & $3.49 \pm 0.06$ & $80.28 \pm 0.09$ & $5.13 \pm 0.13$ & $21.77 \pm 0.62$ \\
\hline & 2 & $19.40 \pm 4.66$ & $257.83 \pm 3.99$ & $8.70 \pm 0.57$ & $10.29 \pm 2.07$ & $6.91 \pm 1.69$ & $0.344 \pm 0.002$ & $3.64 \pm 0.08$ & $79.22 \pm 0.26$ & $4.92 \pm 0.07$ & $19.30 \pm 0.60$ \\
\hline & 3 & $17.66 \pm 1.22$ & $252.53 \pm 8.46$ & $8.43 \pm 1.03$ & $10.47 \pm 1.93$ & $6.43 \pm 0.93$ & $0.365 \pm 0.002$ & $3.59 \pm 0.06$ & $78.11 \pm 0.08$ & $4.02 \pm 0.08$ & $17.25 \pm 0.03$ \\
\hline & 4 & $15.80 \pm 1.48$ & $250.30 \pm 8.47$ & $10.94 \pm 0.71$ & $10.2 \pm 0.63$ & $6.47 \pm 1.37$ & $0.387 \pm 0.002$ & $3.65 \pm 0.04$ & $76.42 \pm 0.28$ & $3.43 \pm 0.04$ & $15.67 \pm 0.03$ \\
\hline & 5 & $12.05 \pm 1.23$ & $243.51 \pm 4.16$ & $8.13 \pm 0.09$ & $10.9 \pm 0.62$ & $7.21 \pm 1.45$ & $0.385 \pm 0.004$ & $3.47 \pm 0.12$ & $75.21 \pm 0.27$ & $3.38 \pm 0.02$ & $15.44 \pm 0.03$ \\
\hline & 6 & $10.34 \pm 0.89$ & $238.61 \pm 0.83$ & $9.17 \pm 0.17$ & $10.32 \pm 1.65$ & $6.65 \pm 0.68$ & $0.386 \pm 0.003$ & $3.62 \pm 0.06$ & $74.07 \pm 0.14$ & $3.38 \pm 0.02$ & $15.34 \pm 0.20$ \\
\hline
\end{tabular}

aw: water activity; $b^{*}$ : the yellow/blue coordinate; $c^{*}$ : the red/green coordinate.

Table 2. Experiment results in storage with vacuum (V).

\begin{tabular}{|c|c|c|c|c|c|c|c|c|c|c|c|}
\hline $\begin{array}{c}\text { Tempera- } \\
\text { ture } \\
{ }^{\circ} \mathrm{C}\end{array}$ & $\begin{array}{l}\text { Time } \\
\text { month }\end{array}$ & $\begin{array}{c}\text { Vitamin C } \\
\mathrm{mg} / 100 \mathrm{~g}\end{array}$ & $\begin{array}{l}\text { Folic Acid } \\
\mathrm{mcg} / 100 \mathrm{~g}\end{array}$ & $\begin{array}{c}\text { Iron } \\
\mathrm{mg} / 100 \mathrm{~g}\end{array}$ & $\begin{array}{l}\text { Dietary } \\
\text { fiber } \\
\mathrm{g} / 100 \mathrm{~g}\end{array}$ & $\begin{array}{l}\text { Protein } \\
\mathrm{g} / \mathbf{1 0 0 g}\end{array}$ & $a_{w}$ & $\mathrm{pH}$ & $\mathrm{L}^{*}$ & $a^{*}$ & $\mathbf{b}^{*}$ \\
\hline \multirow{7}{*}{4} & 0 & $36.68 \pm 0.05$ & $276.92 \pm 3.02$ & $10.26 \pm 0.20$ & $10.2 \pm 1.37$ & $7.03 \pm 1.27$ & $0.277 \pm 0.005$ & $3.65 \pm 0.05$ & $81.25 \pm 0.15$ & $4.21 \pm 0.04$ & $22.59 \pm 0.07$ \\
\hline & 1 & $36.59 \pm 1.42$ & $277.61 \pm 6.22$ & $8.96 \pm 0.40$ & $10.04 \pm 1.17$ & $7.19 \pm 1.15$ & $0.284 \pm 0.002$ & $3.57 \pm 0.02$ & $81.38 \pm 0.38$ & $4.12 \pm 0.06$ & $21.87 \pm 0.11$ \\
\hline & 2 & $32.70 \pm 2.00$ & $275.72 \pm 0.57$ & $11.17 \pm 1.60$ & $10.54 \pm 1.7$ & $6.57 \pm 2.28$ & $0.296 \pm 0.002$ & $3.41 \pm 0.08$ & $79.09 \pm 0.06$ & $4.73 \pm 0.04$ & $22.25 \pm 0.08$ \\
\hline & 3 & $29.31 \pm 1.58$ & $275.06 \pm 7.32$ & $7.57 \pm 0.17$ & $10.34 \pm 2.46$ & $7.15 \pm 1.97$ & $0.313 \pm 0.002$ & $3.61 \pm 0.04$ & $78.71 \pm 0.46$ & $5.09 \pm 0.33$ & $20.11 \pm 0.73$ \\
\hline & 4 & $27.86 \pm 1.01$ & $272.74 \pm 6.20$ & $11.17 \pm 0.21$ & $10.47 \pm 1.68$ & $7.06 \pm 2.01$ & $0.333 \pm 0.002$ & $3.57 \pm 0.12$ & $79.55 \pm 0.18$ & $4.39 \pm 0.19$ & $21.28 \pm 0.10$ \\
\hline & 5 & $26.69 \pm 0.59$ & $273.21 \pm 6.95$ & $9.30 \pm 1.20$ & $10.49 \pm 2.78$ & $7.02 \pm 1.67$ & $0.332 \pm 0.003$ & $3.61 \pm 0.12$ & $80.78 \pm 0.47$ & $4.15 \pm 0.07$ & $21.97 \pm 0.28$ \\
\hline & 6 & $25.83 \pm 5.44$ & $272.39 \pm 6.42$ & $9.77 \pm 1.11$ & $10.08 \pm 1.25$ & $7.26 \pm 1.70$ & $0.333 \pm 0.003$ & $3.68 \pm 0.07$ & $80.04 \pm 0.29$ & $4.29 \pm 0.03$ & $22.13 \pm 0.28$ \\
\hline \multirow{7}{*}{20} & 0 & $36.68 \pm 0.05$ & $276.92 \pm 3.02$ & $10.26 \pm 0.20$ & $10.25 \pm 1.64$ & $6.69 \pm 0.67$ & $0.277 \pm 0.005$ & $3.69 \pm 0.04$ & $81.25 \pm 0.15$ & $4.21 \pm 0.04$ & $22.59 \pm 0.07$ \\
\hline & 1 & $32.32 \pm 1.19$ & $277.65 \pm 12.85$ & $9.52 \pm 0.75$ & $10.73 \pm 3.07$ & $6.38 \pm 0.55$ & $0.342 \pm 0.002$ & $3.55 \pm 0.07$ & $80.13 \pm 0.21$ & $4.11 \pm 0.07$ & $21.09 \pm 0.71$ \\
\hline & 2 & $26.73 \pm 1.25$ & $274.95 \pm 9.38$ & $8.86 \pm 1.08$ & $10.93 \pm 1.73$ & $6.32 \pm 1.82$ & $0.352 \pm 0.002$ & $3.51 \pm 0.08$ & $79.59 \pm 0.02$ & $5.11 \pm 0.64$ & $20.15 \pm 0.06$ \\
\hline & 3 & $25.95 \pm 0.88$ & $273.77 \pm 9.59$ & $10.07 \pm 0.59$ & $10.16 \pm 2.97$ & $6.71 \pm 2.04$ & $0.372 \pm 0.002$ & $3.54 \pm 0.09$ & $78.42 \pm 0.18$ & $4.20 \pm 0.32$ & $19.30 \pm 0.09$ \\
\hline & 4 & $24.16 \pm 1.58$ & $269.48 \pm 7.44$ & $9.88 \pm 0.64$ & $10.59 \pm 1.7$ & $7.18 \pm 1.7$ & $0.394 \pm 0.003$ & $3.61 \pm 0.06$ & $78.48 \pm 0.26$ & $5.52 \pm 0.09$ & $21.01 \pm 0.20$ \\
\hline & 5 & $22.66 \pm 1.59$ & $264.95 \pm 5.14$ & $8.10 \pm 0.14$ & $10.63 \pm 1.63$ & $6.71 \pm 0.83$ & $0.390 \pm 0.003$ & $3.56 \pm 0.06$ & $80.04 \pm 0.29$ & $5.46 \pm 0.03$ & $20.30 \pm 0.04$ \\
\hline & 6 & $22.89 \pm 1.57$ & $263.22 \pm 8.07$ & $8.87 \pm 0.48$ & $10.31 \pm 1.62$ & $6.48 \pm 0.84$ & $0.393 \pm 0.002$ & $3.57 \pm 0.03$ & $79.23 \pm 0.29$ & $5.49 \pm 0.04$ & $20.31 \pm 0.26$ \\
\hline \multirow{7}{*}{30} & 0 & $36.68 \pm 0.05$ & $276.92 \pm 3.02$ & $10.26 \pm 0.20$ & $10.11 \pm 2.89$ & $7.04 \pm 1.64$ & $0.277 \pm 0.005$ & $3.47 \pm 0.07$ & $81.25 \pm 0.15$ & $4.21 \pm 0.04$ & $22.59 \pm 0.07$ \\
\hline & 1 & $29.17 \pm 2.08$ & $271.28 \pm 10.75$ & $9.06 \pm 0.70$ & $10.05 \pm 2.04$ & $6.48 \pm 1.10$ & $0.333 \pm 0.002$ & $3.63 \pm 0.03$ & $81.81 \pm 0.17$ & $4.21 \pm 0.04$ & $21.08 \pm 0.10$ \\
\hline & 2 & $23.79 \pm 0.89$ & $264.45 \pm 4.88$ & $8.27 \pm 0.17$ & $10.03 \pm 3.29$ & $6.96 \pm 0.79$ & $0.342 \pm 0.002$ & $3.58 \pm 0.09$ & $80.18 \pm 0.15$ & $5.03 \pm 0.06$ & $18.33 \pm 0.11$ \\
\hline & 3 & $19.73 \pm 0.74$ & $262.09 \pm 3.45$ & $9.37 \pm 0.52$ & $10.64 \pm 1.74$ & $7.14 \pm 1.89$ & $0.363 \pm 0.002$ & $3.49 \pm 0.02$ & $79.03 \pm 0.04$ & $5.16 \pm 0.07$ & $17.22 \pm 0.07$ \\
\hline & 4 & $17.09 \pm 0.99$ & $259.96 \pm 2.65$ & $9.63 \pm 0.21$ & $10.67 \pm 2.45$ & $7.16 \pm 0.84$ & $0.383 \pm 0.002$ & $3.53 \pm 0.03$ & $79.72 \pm 0.05$ & $5.20 \pm 0.07$ & $17.34 \pm 0.06$ \\
\hline & 5 & $13.47 \pm 0.96$ & $253.50 \pm 0.30$ & $8.10 \pm 0.08$ & $10.9 \pm 1.42$ & $7.21 \pm 1.86$ & $0.382 \pm 0.004$ & $3.59 \pm 0.12$ & $79.72 \pm 0.70$ & $5.17 \pm 0.02$ & $17.41 \pm 0.22$ \\
\hline & 6 & $11.64 \pm 0.53$ & $248.38 \pm 6.54$ & $10.60 \pm 2.51$ & $10.73 \pm 1.61$ & $7.11 \pm 0.92$ & $0.383 \pm 0.003$ & $3.43 \pm 0.06$ & $79.23 \pm 0.29$ & $5.22 \pm 0.02$ & $17.33 \pm 0.22$ \\
\hline
\end{tabular}


Table 3. Statistical results to the cape gooseberry powder.

\begin{tabular}{|c|c|c|c|c|c|c|c|c|c|c|c|c|c|c|}
\hline \multirow[b]{2}{*}{$\mathrm{N}^{\circ}$} & \multirow[b]{2}{*}{ Interaction } & \multicolumn{13}{|c|}{ NV } \\
\hline & & $\begin{array}{c}\text { Vitamin } \\
\text { C }\end{array}$ & $\begin{array}{l}\text { Folic } \\
\text { Acid }\end{array}$ & Iron & \multicolumn{2}{|l|}{$a_{w}$} & \multicolumn{2}{|c|}{$a^{*}$} & $b^{*}$ & $\mathrm{C}$ & h & Protein & Fiber & $\mathrm{pH}$ \\
\hline A & Time (month) & $\underline{S}$ & $\underline{\mathrm{S}}$ & $\underline{\mathrm{S}}$ & $S$ & $\$$ & \multicolumn{2}{|r|}{$\underline{S}$} & $\underline{S}$ & $\underline{S}$ & $\underline{S}$ & NS & NS & NS \\
\hline B & $\begin{array}{c}\text { Temperature } \\
\left({ }^{\circ} \mathrm{C}\right)\end{array}$ & $\underline{\mathrm{S}}$ & $\underline{\mathrm{S}}$ & NS & S & $£$ & \multicolumn{2}{|r|}{ S } & $\underline{S}$ & $\underline{\mathrm{S}}$ & $\underline{\mathrm{S}}$ & NS & NS & NS \\
\hline$A^{2}$ & Interaction $^{2}$ & $\underline{\mathrm{S}}$ & $\underline{\mathrm{S}}$ & NS & $\underline{S}$ & 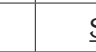 & \multicolumn{2}{|r|}{$\underline{S}$} & $\underline{S}$ & $\underline{\mathrm{S}}$ & $\mathrm{S}$ & NS & NS & NS \\
\hline$B^{2}$ & Interaction $^{2}$ & $\mathrm{~S}$ & NS & NS & $\underline{\mathrm{S}}$ & $N$ & \multicolumn{2}{|r|}{$\underline{S}$} & $\mathrm{~S}$ & $\mathrm{~S}$ & $\mathrm{~S}$ & NS & NS & NS \\
\hline$A B$ & $\begin{array}{l}\text { Interaction } \\
\text { among factors }\end{array}$ & $\underline{\mathrm{S}}$ & $\underline{\mathrm{S}}$ & NS & \multicolumn{2}{|l|}{ S } & \multicolumn{2}{|r|}{$\underline{\mathrm{S}}$} & $\underline{\mathrm{S}}$ & $\underline{\mathrm{S}}$ & NS & NS & NS & NS \\
\hline $\mathrm{R}^{2}$ & ---- & 0.913 & 0.974 & 0.112 & 0.927 & 0.9 & \multicolumn{2}{|c|}{0.958} & 0.915 & 0.908 & 0.962 & 0.151 & 0.109 & 0.102 \\
\hline $\begin{array}{l}\text { Statistical } \\
\text { Signifi- } \\
\text { cance }\end{array}$ & ----- & $S$ & $S$ & NS & S & $\$$ & \multicolumn{2}{|r|}{ S } & S & $S$ & S & NS & NS & NS \\
\hline \multirow[b]{2}{*}{$\mathrm{N}^{\circ}$} & \multirow[b]{2}{*}{ Interaction } & \multicolumn{13}{|c|}{$\mathbf{V}$} \\
\hline & & \multicolumn{2}{|c|}{ Vitamin C } & $\begin{array}{l}\text { Folic } \\
\text { Acid }\end{array}$ & Iron & $a_{w}$ & $\mathrm{~L}^{*}$ & $a^{*}$ & $\mathbf{b}^{*}$ & C & h & Protein & Fiber & $\mathrm{pH}$ \\
\hline A & Time (month) & \multicolumn{2}{|c|}{$\underline{\mathrm{S}}$} & $\underline{\mathrm{S}}$ & NS & S & NS & S & $\underline{S}$ & $\underline{\mathrm{S}}$ & $\underline{S}$ & NS & NS & NS \\
\hline B & $\begin{array}{c}\text { Temperature } \\
\left({ }^{\circ} \mathrm{C}\right)\end{array}$ & \multicolumn{2}{|c|}{$\underline{S}$} & $\underline{\mathrm{S}}$ & NS & $S$ & $\underline{\mathrm{S}}$ & S & $\underline{\mathrm{S}}$ & $\underline{\mathrm{S}}$ & $\underline{S}$ & NS & NS & NS \\
\hline$A^{2}$ & Interaction $^{2}$ & \multicolumn{2}{|c|}{$\underline{S}$} & $\underline{\mathrm{S}}$ & NS & $\underline{S}$ & $S$ & $\underline{S}$ & $\underline{S}$ & $\underline{S}$ & NS & NS & NS & NS \\
\hline $\mathrm{B}^{2}$ & Interaction $^{2}$ & \multicolumn{2}{|c|}{$\mathrm{S}$} & NS & NS & $\underline{S}$ & $\mathrm{~s}$ & $\underline{S}$ & $S$ & $S$ & $\mathrm{~s}$ & NS & NS & NS \\
\hline$A B$ & $\begin{array}{c}\text { Interaction } \\
\text { among factors }\end{array}$ & \multicolumn{2}{|c|}{$\underline{\mathrm{S}}$} & $\underline{\mathrm{S}}$ & NS & $S$ & $\underline{\mathrm{S}}$ & S & $\underline{\mathrm{S}}$ & $\underline{\mathrm{S}}$ & $\underline{\mathrm{S}}$ & NS & NS & NS \\
\hline $\mathrm{R}^{2}$ & ---- & \multicolumn{2}{|c|}{0.917} & 0.991 & 0.033 & 0.945 & 0.905 & 0.957 & 0.987 & 0.957 & 0.938 & 0.138 & 0.178 & 0.144 \\
\hline $\begin{array}{c}\text { Statistical } \\
\text { Signifi- } \\
\text { cance }\end{array}$ & ---- & \multicolumn{2}{|c|}{$S$} & $S$ & NS & $S$ & $S$ & $S$ & $S$ & $S$ & $S$ & NS & NS & NS \\
\hline
\end{tabular}

NS: Not significant; S: Significant to a value $\mathrm{p}<0.05$; aw: water activity; $\mathrm{b}^{*}$ : the yellow/blue coordinate; $\mathrm{c}^{*}$ : the red/green coordinate. $\mathrm{h}$ : cylindrical coordinate (hue). C: cylindrical coordinate (chroma).

The vitamin $C$ tends to decrease, thus the storage temperature was an important factor, gaining significance with increase of temperature and storage time. Increasing the temperature raises the water mobility inside the powder particle favoring degradative reactions (Rodríguez et al. 2009). Vitamin C is an unstable and weak vitamin because of the high reactivity of the enediol structure. Its main degradation route is oxidation to dehydroascorbic acid, which tends to suffer a series of reactions with amino acids and acids deriving into active formation of pigments (Cernisev, 2010). The kinetic loss of vitamin $\mathrm{C}$ was lower at $4^{\circ} \mathrm{C}$, since low temperatures have cryprotectant effects. This adds to the greater attraction with maltodextrin by molecular affinity, having common $\mathrm{OH}$ groups which enable high interaction (Dib Taxi et al. 2003). Studies have demonstrated that vitamin $\mathrm{C}$ contents decrease during storage depending on temperature, time and condition of package (Hymavathi \& Khader, 2005; Lisiewska \& Kmiecik, 2000).

Regarding folic acid, individual factors and quadratic interactions contribute to the kinetic loss of this PAC, presenting a lower sensitivity in the first 2 months and becoming more critical at temperature of $30^{\circ} \mathrm{C}$. The degradation of folic acid is much less than that of vitamin C, due to the fact that its chemical nature possesses extreme carbonyl and amino groups, enabling them to interact with the soy protein and the maltodextrin binding strongly to these compounds and favoring protection against storage temperature (Krishnam et al. 2005). These results agree with similar studies by Cai \& Corke (2000) and Quek et al. (2007). 

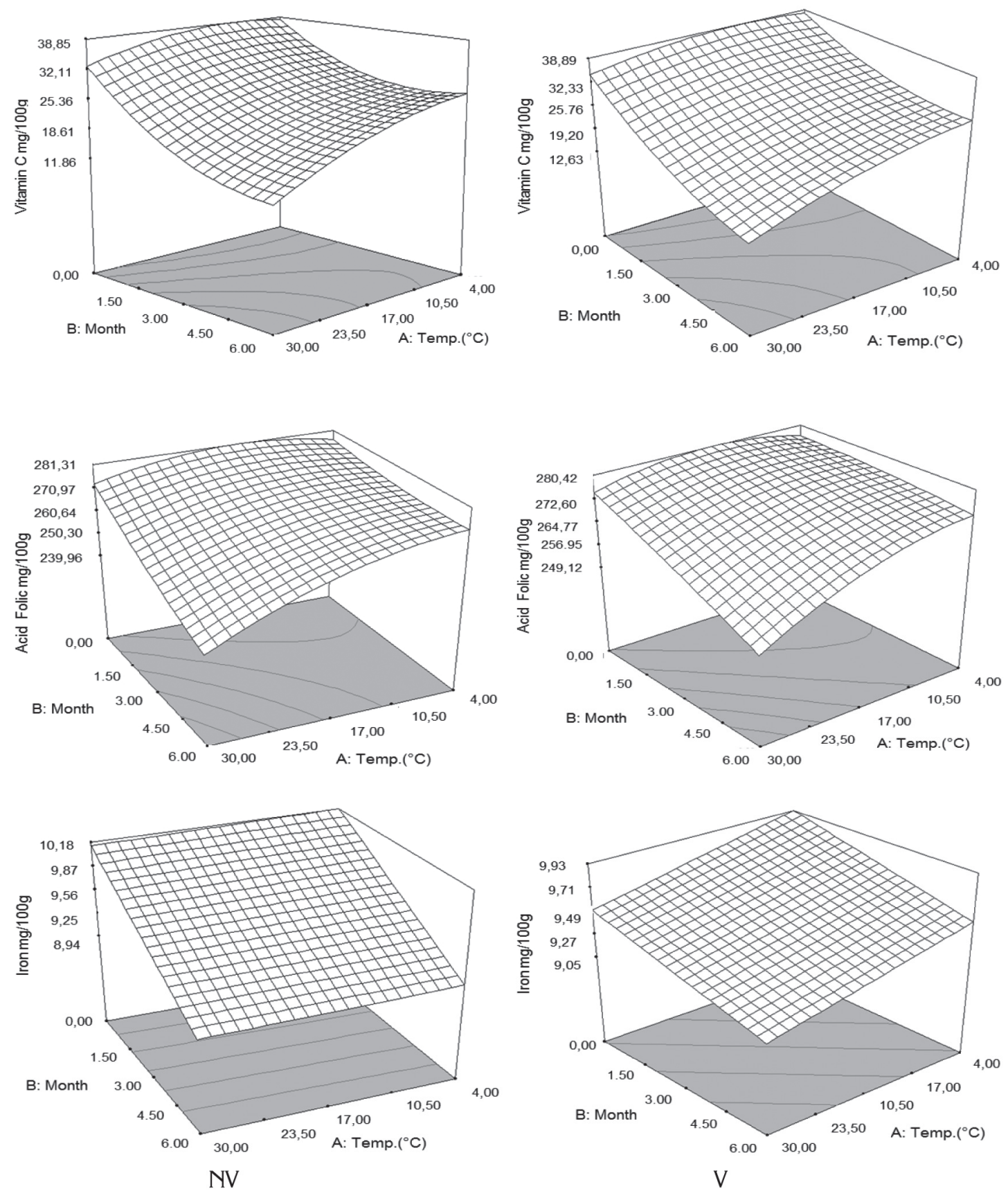

Figure 1. Response surface of the contents of vitamin C, vitamin $\mathrm{B}_{9}$, and iron for each $100 \mathrm{~g}$ of cape gooseberry powder: without vacuum (NV) and with vacuum (V).

Iron in cape gooseberry only presented significant differences with regards to the factor of storage time $(p<0.05)$. However, these changes are not very perceivable. Iron is a nonthermosensitive mineral (Krishnam et al. 2005) and the results show that factors as temperature and packaging conditions have no statistical effects and are favored by the protective effect given by maltodextrin. Similar studies have been reported by other products such as green barley powder (García et al. 2004).

Protein and dietary fiber contents did not present significant differences regarding the storage time and storage temperature factors. This is attributed to the fact that they are stable macromolecular compounds to conditions of low 
water activity $\mathrm{a}_{\mathrm{w}}$ (Sudha et al. 2007). The protein contained in the cape gooseberry powder can interact with iron through the amino and carbonyl groups by means of hydrogen bridges. This situation is favored by the $\mathrm{pH}$ of the fruits pulp of 3.4 0.1 (García et al. 2004) improving its protection through the storage time. Besides, the aminochelated chemical structure of iron bisglycinate of high molecular weight, allows binding to formulation components such as soy protein, generating a more resistant structure for the encapsulated compounds and reducing iron loss (Oliveira et al. 2007). Dietary fiber and protein contents averages were approximately $6.89 \pm 0.27$, and $10.44 \pm 0.29 \mathrm{~g} / 100 \mathrm{~g}$ cape gooseberry powder, respectively. These correspond to $13.8 \%$ of the daily reference value (DRV) of protein, and to $41.8 \%$ DRV of dietary fiber, in accordance to the nutritional requirements established by Colombian Government.
The contents of vitamin $\mathrm{C}, \mathrm{B}_{9}$ and iron achieved at the end of the storage over a portion of $100 \mathrm{~g}$ of cape gooseberry powder expressed as \% DRV enable identifying the product in the Colombian standard framework, at 4 and $20^{\circ} \mathrm{C}$ with the descriptor of "Excellent source of vitamin C, folic acid, iron and dietary fiber" and "Good source of protein." whereas at $30^{\circ} \mathrm{C}$, as "Excellent source of folic acid, iron and dietary fiber" and "Good source of protein and vitamin C".

Cape Gooseberry Powder Properties: Figure 2 shows the surface response of water activity $\left(\mathrm{a}_{\mathrm{w}}\right)$ as a function of the studied factors considering the packaging conditions (NV) and $(\mathrm{V})$. The water activity presented under both packaging conditions significant statistical differences $(p<0.05)$. An increase of aw occurs in relation to storage temperature and storage time.

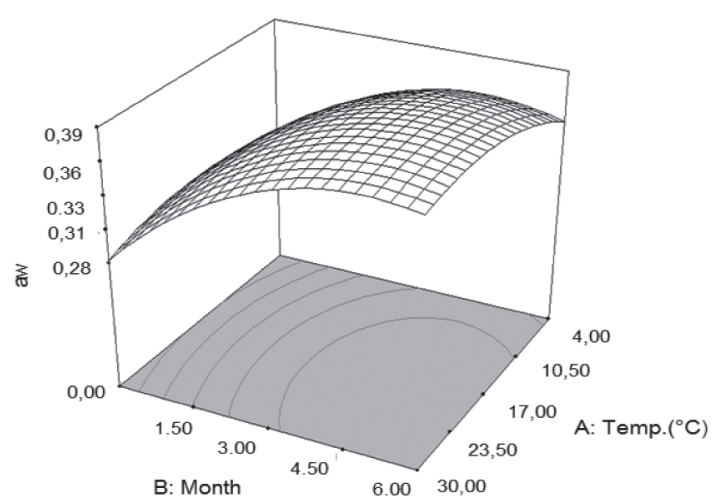

NV

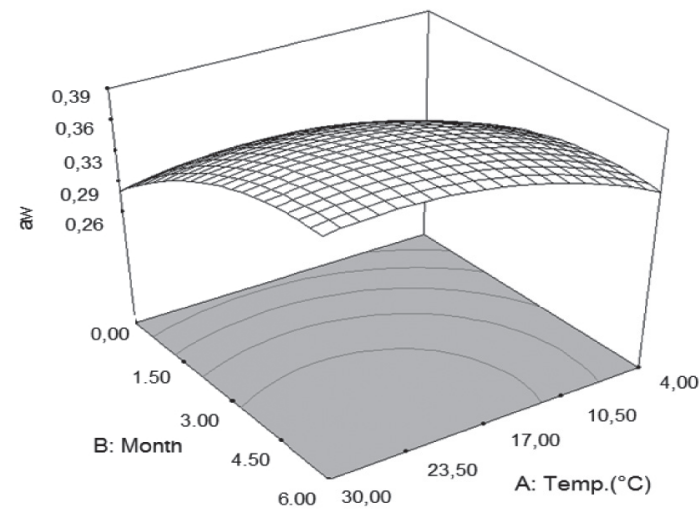

V

Figure 2. Response surface of $a_{w}$ in function of the study factors: without vacuum (NV) and with vacuum (V).

The gain in water vapor depends on the permeability of the low density polyethylene packaging and its interaction with temperature due to the variation of the relative humidity of the air surrounding the packaging plus the hygroscopicity offered by the product (Tonon et al. 2008). The water activity presented the lowest variations at $4^{\circ} \mathrm{C}$, whereas at higher temperatures it remains stable from the third to the sixth month. This situation of equilibrium in aw is favored by maltodextrin, a material of low hygroscopicity which presents a good efficiency acting as a protective agent against humidity (Rodríguez-Hernández et al. 2005). A similar study presented by Cai \& Corke (2000) working with spray drying in betacyanin pigments where was finding a reduction of hygroscopicity close to $20 \%$ at higher concentrations of maltodextrin.
Figure 3 presents the response surface of the color parameters $\mathrm{L}, \mathrm{a}$ and $\mathrm{b}$ in function of the study factors. All parameters presented significant statistical differences $(p<0.05)$ regarding storage temperature $(A)$, storage time (B) and interactions ( $\mathrm{AB}$ ) (Table 3). A diminishing trend was observed in the color parameters during storage time, being more distinct the higher the storage temperature. However, despite the statistical differences, the changes of parameters at 4 and $20^{\circ} \mathrm{C}$ are not very noticeable in any of the packaging conditions (L: $81.5 \rightarrow 78.0$; a: $5.5 \rightarrow 4.0$; b: $23.0 \rightarrow 19.0$ ) whereas at $30^{\circ} \mathrm{C}$ mainly noticeable are the changes in luminosity L $(81.5 \rightarrow 74.0)$ and chromaticity b $(23.0 \rightarrow 15.0)$. 

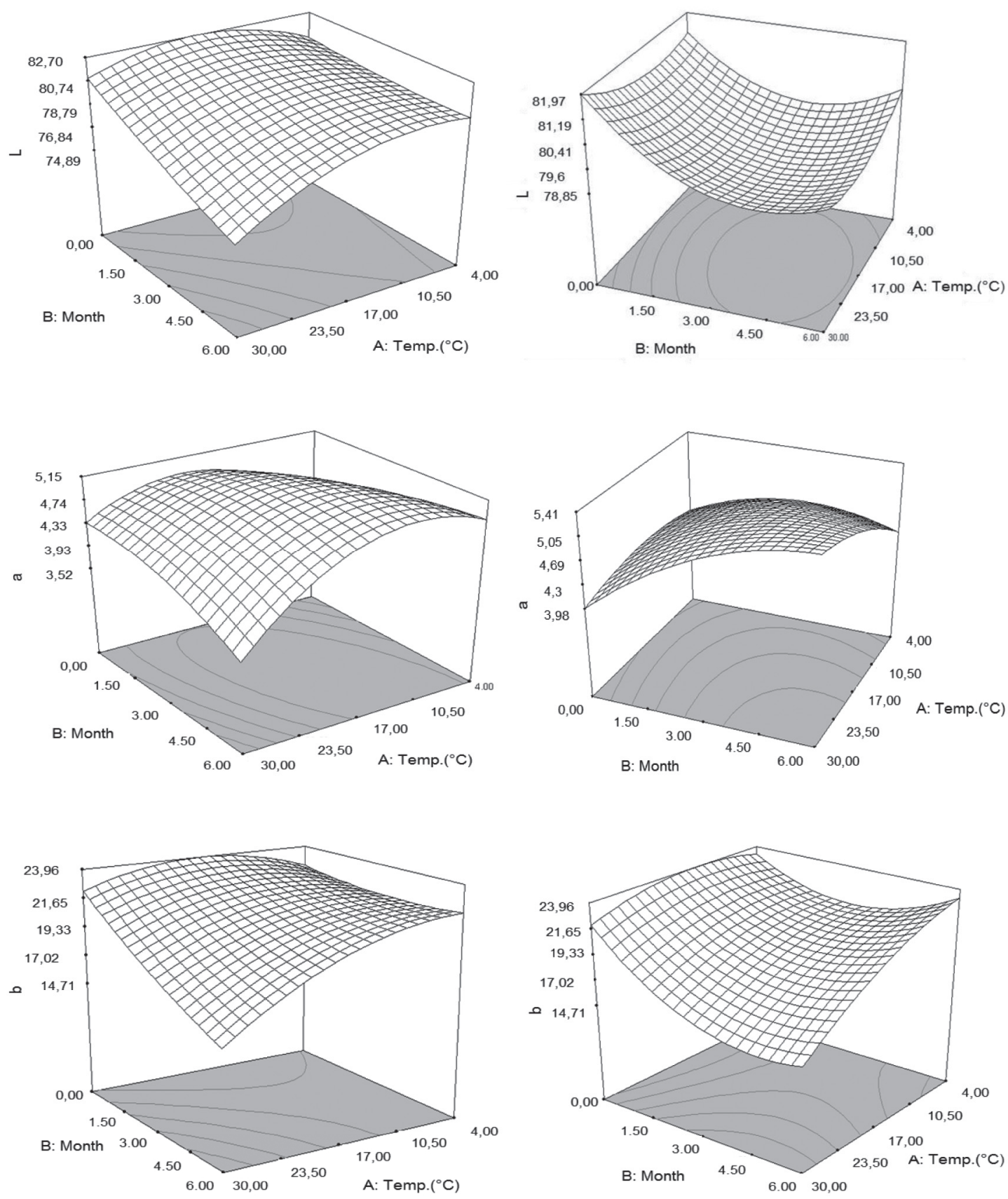

V

NV

Figure 3. Color parameters by cartesian coordinates (CIE-Lab) of the cape gooseberry powder with vacuum (V) and without vacuum (NV).

The decrease of luminosity $L$ at high storage temperatures with the time, reflects the darkening of the particles surface, which can be mainly attributable to non-enzymatic reactions (Urbanyi \& Horti, 1989); while the decrease of the chromaticity $\mathrm{a}$ and $\mathrm{b}$ with time and temperature, reflects the degradation of carotenoid pigments (Ruíz et al. 2005). This situation produces that in the chromatic plane $a b$, the samples displace towards the interior of the area of grays decreasing the saturation or chroma $(C)(\cong 24 \AA 15)$ and hue $(h)\left(\cong 80^{\circ} \circledR\right.$ $73^{\circ}$ ). Similar changes have been observed during the storage of tomato powder (Fengxia et al. 2010). This situation makes the samples take a more orange color, stronger in the CV 
samples than in the SV due to the mechanical compacting exerted by the vacuum in the samples. Similar results were found by Cortés et al. (2008) in orange juice and by Arias et al. (2000) in tomato.

According to the results of this research it can be concluded that during the storage of cape gooseberry powder added with vitamin C, iron, folic acid, soluble fiber (polydextrose) and soy protein, there is an influence of the storage temperature and storage time on the product, while packaging condition is not a significant factor. Vitamin $\mathrm{C}$ and folic acid contents were the properties most affected with the increase of storage temperature and time; while iron (ferrous bisglycinate), protein and dietary fiber did not present noticeable changes.

Additionally, a protective effect of maltodextrin as an encapsulating agent was observed. The results disclosed that product storage at a temperature between 4 and $20^{\circ} \mathrm{C}$, packaged without vacuum permits labeling the product, using the Colombian standard framework, during the 6 months of storage with the descriptor of "Excellent source of vitamin C, folic acid, iron and dietary fiber" and "Good source of protein".

Conflict of interests: The manuscript was prepared and reviewed with the participation of the authors, who declare that there exists no conflict of interest that puts in risk the validity of the results presented.

\section{BIBLIOGRAPHY}

1. AOAC-ASSOCIATION OF OFFICIAL ANALYTICAL CHEMISTS. 1990. Official Methods of Analysis of AOAC International. 15th edition. Arlington, VA. 1018p.

2. AOAC-ASSOCIATION OF OFFICIAL ANALYTICAL CHEMISTS. 1996. Official Methods of Analysis. Basic calculations for chemical and biological analyses. Arlington, VA. 135p.

3. AOAC-ASSOCIATION OF OFFICIAL ANALYTICAL CHEMISTS. 1998. Official Methods of Analysis of AOAC International.16th edition. Gaithersburg, MD. 1100p.

4. ARCOT, J.; SHRESTHA, A. 2005. Folate: methods of analysis. Trends Food Sci. \& Techn. 16(6):253-266.

5. ARIAS, R.; LEE, T.C.; LOGENDRA, L.; JANES, H. 2000. Correlation of lycopene measured by HPLC with the $\mathrm{L}^{*}, \mathrm{a}^{*}, \mathrm{~b}$ * color readings of a hydroponic tomato and the relationship of maturity with color and lycopene content. J. Agr. Food Chem. 48(5):1697-1702.
6. BHANDARI, B.R.; SENOUSSI, A.; DUMOULIN, E.D.; LEBERT, A. 1993. Spray drying of concentrated fruit juices. Drying Techn. 11(5):1081-1092.

7. CAI, Y.Z.; CORKE, H. 2000. Production and properties of spray-dried Amaranthus betacyanin pigments. J. Food Sci. 65(6):1248-1252.

8. CERNISEV, S. 2010. Effects of conventional and multistage drying processing on nonenzymatic browning in tomato. J. Food Eng. 96(1):114-118.

9. CORTÉS, M. 2012. Ingeniería de matrices: una herramienta efectiva para la generación de valor agregado en alimentos porosos. Vitae. 19(2):S39-S40.

10. CORTÉS, C.; ESTEVE, M.J.; FRIGOLA, A. 2008. Color of orange juice treated by high intensity pulsed electric fields during refrigerated storage and comparison with pasteurized juice. J. Food Control. 19(2):15158.

11. DHIRMAN, T.R.; ANAN, G.R.; SATTER, L.D.; PARIZA, M.W. 1999. Conjugated linoleico acid content of milk from cows fed different diets. J. Dairy Sci. 82 (10):2146-2156.

12. DIB TAXI, C.M.; MENEZES, H.C.; SANTOS, A.B.; GROSSO, C.R. 2003. Study of the microencapsulation of camu-camu (Myrciaria dubia) juice. J. Microencapsulation. 20(4):443-448.

13. DOLINSKY, A.; MALETSKAYA, K.; SNEZHKIN, Y. 2000. Fruit and vegetable powders production on the bases of spray and convective drying methods. Drying Techn. 18(3):747-758.

14. ERSUS, S.; YURDAGEL, U. 2007. Microencapsulation de anthocyanin pigments of black carrot (Daucus carota L.) by spray drier. J. Food Eng. 80(3):805812.

15. FAZAELI, M.; EMAM-DJOMEH, Z.; KALBASI ASHTARI, A.; OMID, M. 2012. Effect of spray drying conditions and feed composition on the physical properties of black mulberry juice powder. Food Biop. Proce. 90(4):667-675

16. FENGXIA, L.; XIAMIN, C.: HOUYIN, W.; XIAOJUN, L. 2010. Changes of tomato powder qualities during storage. Powder Techn. 204(1):159-166. 
17. GARCÍA, C.; GONZÁLEZ, M.; OCHOA, L.; MEDRANO, H. 2004. Microencapsulación de jugo de cebada verde mediante secado por atomización. Cien. Tecn. Aliment. 4(4):262-266.

18. GIBSON, R.S. 1997. Technological approaches to combatting iron deficiency. J. Clinic Nutr. 51(4):2527.

19. GUTIÉRREZ, T.; HOYOS, O.; PÁEZ, M. 2007. Determinación del contenido de ácido ascórbico en uchuva (Physalis peruviana L.) por cromatografía líquida de alta resolución (HPLC.) Rev. Biotecn. Sector Agropec. Agroindust. 5(1):70-79.

20. HEUDI, O.; KILINÇ, T.; FONTANNAZ, P. 2005. Separation of water-soluble vitamins by reversed-phase high performance liquid chromatography with ultra-violet detection: Application to polyvitaminated premixes. J. Chromatogr. 1070(2):49-56.

21. HYMAVATHI, T.V.; KHADER, V. 2005. Carotene, ascorbic acid and sugar content of vacuum dehydrated ripe mango powders stored in flexible packaging material. J. Food Comp. Anal. 18(2-3):181-192.

22. ICONTEC. 1999. Instituto Colombiano de Normas Técnicas y Certificación. Frutas frescas: Uchuva. Especificaciones. Norma Técnica Colombiana NTC 4580. 15p.

23. ICONTEC. 1999. Instituto Colombiano de Normas Técnicas y Certificación. Método de determinación del contenido de nitrógeno en alimentos para animales por el proceso kjeldahl y un método del cálculo del contenido de proteína cruda. Norma Técnica Colombiana NTC 4657. 11p.

24. ICONTEC. 2000. Instituto Colombiano de Normas Técnicas y Certificación. Productos de frutas y verduras. Determinación del contenido de cobre. Método espectrométrico de absorción atómica de llama. Norma Técnica Colombiana NTC 4807. 8p.

25. KOSHIZAKA, T.; NISHIKIMI, M.; OZAWA, T. Y.; YAGI, K. 1988. Isolation sequence analysis of a complementary ADN encoding rat liver L-gulono-gammalactonaoxidasa, a key enzima for L-ácido ascórbico biosynthesis. J. Biol. Chemi. 263(1):1619-1621.

26. KRISHNAM, S.; KSHIRSAGAR, A.C.; SINGHAL R. S. 2005. The use of gum arabic and modified starch in the microencapsulation of a food flavoring agent. J. Carbohydr. Polym. 62(4):309-315.

27. KUDRA, T.; MUJUMDAR, A.S. 2012. Advanced Drying Technologies, Second Edition, CRC Press, New York. 438p.

28. LISIEWSKA, Z.; KMIECIK, W. 2000. Effect of storage period and temperature on the chemical composition and organoleptic quality of frozen tomato cubes. Food Chem. 70(2):167-173.

29. MAZORRA, M.; QUINTANA, A.; MIRANDA, D.; FISCHER, G.; CHAPARRO, M. 2006. Aspectos anatómicos de la formación y crecimiento del fruto de uchuva Physalis peruviana (Solanaceae). Acta Biol. Col. 11(1):69-81.

30. OLIVEIRA,A.C.; MORETTI, T.S.;BOSCHINI, C.;BALIERO, J.C.C.; FREITAS, O.; FAVARO-TRINDADE, C.S. 2007. Stability of microencapsulated B. lactis (BI 01) and L. acidophilus (LAC 4) by complex coacervation followed by spray drying. J. Microencap. 24(7):685693.

31. OSORIO, D.; ROLDÁN, J.C. 2004. Manual de la uchuva Grupo Latino LTDA. 85p.

32. PEÑA, R.F.; CORTÉS, M.; MONTOYA, O.I. 2013. Evaluation of the physicochemical, physical and sensory properties of fresh cape gooseberry and vacuum impregnated with physiologically active components. Vitae. 20(1):13-22.

33. PÉREZ, S.; CANCHO, B.; GARCÍA, S.; SIMAL, J. 2006. Screening for folic acid content in vitamin-fortified beverages. J. Food Control. 17(11):900-904.

34. PRESCHA, A.; BIERNAT, J.; WEBER, R.; ZUK, M.; SZOPA, J. 2003. The influence of modified 14-3-3 protein synthesis in potato plants on the nutritional value of the tubers. Food Chem. 82(4):611-617.

35. PRONCZUK, A.; HAYES, K.C. 2006. Hypocholesterolemic effect of dietary polydextrose in gerbils and humans. Nutr. Res. 26(1):27-31.

36. PUENTE, L.A.; PINTO-MUÑOZ, C.A.; CASTRO, E.S.; CORTÉS, M. 2011. Physalis peruviana Linneaus, the multiple properties of a highly functional fruit: A review. Food Res. Int. 44(7):1733-1740. 
37. QUEK, S.Y.; CHOK, N.K.; SWEDLUND, P. 2007. The physicochemical properties of spray dried watermelon powder. J. Chem. Eng. Proc. 46(5):386392.

38. RIBEIRO, C.; ZIMERI, J.E.; YILDIZ, E.; KOKINI, J.L. 2003. Estimation of effective diffusivities and glass transition temperature as a function of moisture content. Carbohydr. Polym. 51:273-280.

39. RODRÍGUEZ, H.; PEIXOTO, K.; JAEGER, L.; RODRÍGUEZ, L.; PEDROSA, C.; TRINDADE, A.; PIERUCCI, R. 2009. Legumes seeds protein isolates in the production of ascorbic acid microparticles. J. Food Res. Int. 42(1):115-121.

40. RODRÍGUEZ, S.L.; ELIKA, M. 2007. Efecto de la ingesta de Physalis peruviana (aguaymanto) sobre la glicemia postprandial en adultos jóvenes. Rev. Méd. Vallejiana. 4(1):43-53.

41. RODRÍGUEZ-HERNÁNDEZ, G.R.; GONZÁLEZ-GARCÍA, R.; GRAJALES-LAGUNES, A.; RUIZ-CABRERA, M.A. 2005. Spray-drying of cactus pear juice (Opuntia streptacantha): effect on the physicochemical properties of powder and reconstituted product. Drying Techn. 23(4):955-973.

42. ROJAS, M., PEÑUELA, A., GÓMEZ, C., ARISTIZABAL, G., CHAPARRO, M.; LÓPEZ, J. 2004. Caracterización de los productos hortofrutícolas colombianos y establecimiento de las normas técnicas de calidad: Cenicafé, SENA, FEDERACAFÉ. p.115-120.

43. RUÍZ, D.; EGEA, J.; TOMÁS-BARBERÁN, F.A.; GIL, M.I. 2005. Carotenoids from new apricot (Prunus armeniaca L.) varieties and their relationship with flesh and skin color. J. Agric. Food. Chem. 53(16):6368-6374.

44. SAÉNZ, C.; TAPIA, S.; CHAVEZ, J.; PAZ, R. 2009. Microencapsulation by spry drying of bioactives compounds from captus pear (Opuntia ficusindica). Food Chem. 114(2):616-622.

45. SUDHA, M.L.; SRIVASTAVA, A.K.; LEELAVATHI, A. 2007. Studies on pasting and structural characteristics of thermally treated wheat germ. Eur. Food Res.Techn. 225(3):351-357.

46. TONON, R.; BRABET, C.; HUBINGER, M. 2008. Influence of process conditions on the physicochemical properties of acai (Euterpe oleraceae Mart.) powder produced by spray drying. J. Food Eng. 88(3):411418.

47. URBANYI, G.; HORTI, K. 1989. Color and carotenoids content of quick-frozen tomato cubes during frozen storage. J. Acta Aliment. 18(3):247-267.

48. WOUN, T.L.; NORDIN IBRAHIM, M.; KAMILB, R.; SALEENA TAIPA, F. 2011. Empirical modeling for spray drying process of sticky and non-sticky products. Procedia Food Sci. 1:690-697

49. XUDONG, Y.; AL-BABILI, S.; KLO ETI, A.; JING, Z.; LUCCA, P.; BEYER, P.; POTRYKUS, I. 2000. Engineering the provitamin A (b-carotene) biosynth etic pathway into (carotenoid-free) rice endosperm. Sci. 287:303-305.

Received: 28 August 2013

Accepted: 11 February 2014

Como citar:

Hernández-Sandoval, G.R.; Cortés-Rodríguez, M.; Ciro-Velásquez H.J. 2014. Effect of storage conditions on quality of a functional powder of cape gooseberry obtained by spray drying. Rev. U.D.C.A Act. \& Div. Cient. 17(1): 139-149. 
